\title{
La durabilité de l'eau agricole en Méditerranée à l'épreuve des enjeux sociétaux contemporains
}

\author{
Chantal Aspe* \\ Marie Jacqué **
}

\section{Sommaire}

Sommaire du Congrès International portant sur les usages écologiques, économiques et sociaux des eaux d'irrigation en Méditerranée, qui s'est tenu 'a l'Université de Provence (Marseille) les 20 et 21 Janvier 2010. II y eu 4 axes de débat ; de l'irrigation à la gestion durable de l'eau, les droits de l'eau (propriété et appropriation symbolique), biodiversité et paysage de l'eau et perspective de gestion, participation et gouvernance. La reconnaissance d'expérience des 2 cotés de la Méditerranée, avec des processus divers et contrastés, a offert aux 200 congressistes un cadre riche en références historiques et actuelles et un registre intéressant de propositions pour le futur.

\section{Mots-clés}

Eau; Irrigation; Durable; Propriété; Paysage; Gestion

\section{Resumen: La sostenibilidad del agua agrícola en el Mediterráneo frente a los retos de las sociedades contemporáneas}

Crónica del Congreso Internacional que sobre Los Usos ecológicos, económicos y sociales del agua de riego en el Mediterráneo, se celebró en la Universidad de Provenza (Marsella) durante los días 20 y 21 de enero de 2010. Cuatro fueron sus ejes de debate: Del regadío a la gestión sostenible del agua, los derechos de agua (propiedad y apropiación simbólica), biodiversidad y paisajes del agua y perspectivas de gestión, participación y gobernanza. El reconocimiento de experiencias de las dos orillas del Mediterráneo, con procesos diversos y contrastados, ofreció a los 200 congresistas un marco muy rico de referencias históricas y actuales y un elenco de interesantes propuestas de futuro.

\section{Palabras clave}

Agua; Regadío; Sostenibilidad; Propiedad; Paisajes; Gestión

\section{Abstract: Sustainability of agricultural water in the Mediterranean vs. contemporary society issues}

The paper is a review of the international conference entitled "Ecological, economic and social uses of agricultural water in the Mediterranean area: What is at stake?" held on January 20-21 at the University of Provence (Marseilles). The seminar was structured around the following four key points: from irrigation to sustainable water management, water rights: ownership and symbolic appropriation, biodiversity and water landscapes, and prospects for management, participation and governance. The analysis and comparison between experiences on both sides of the Mediterranean with different processes gave the 200 participants a rich framework of historic and current references and a range of interesting proposals for the future.

\section{Key words}

Water, irrigation; Sustainability ; Ownership; Landscapes ; Management

Recibido: 05/05/2011; aceptado definitivamente: 14/06/2011

* MCF HDR en Sociologie, Aix-Marseille Université, LPED. E-mail: chantal.aspe@univ-provence.fr

${ }^{* *}$ MCF en Sociologie, Aix-Marseille Université, LPED. E-mail : marie.jacque@univmed.fr 


\section{Compte-rendu du colloque} international Usages écologiques, économiques et sociaux de l'eau agricole en Méditerranée : quels enjeux pour quels services? ${ }^{2}$

Les 20 et 21 janvier 2011, s'est tenu à l'Université de Provence à Marseille (France), un colloque international et interdisciplinaire intitulé : "Usages écologiques, économiques et sociaux de l'eau agricole en Méditerranée : quels enjeux pour quels services ?". Trois laboratoires de recherche ont participé à son élaboration et organisation : le LPED (Laboratoire Population Environnement Développement, UMR IRD), l'IMEP (Institut Méditerranéen d'Ecologie et de Paléoécologie, UMR CNRS) situés à Marseille et le GIEST (Grupo de Investigacion Estructuras y Sistemas Territoriales) de Séville. II a regroupé plus de 200 chercheurs en sciences sociales, sciences de la nature et sciences de l'ingénieur, ainsi que des professionnels de la gestion de l'eau du bassin méditerranéen. Six pays étaient représentés : l'Espagne, l'Italie, le Maroc, l'Algérie, la Tunisie et la France. C'est le premier colloque international dédié aux enjeux et perspectives de la gestion de l'eau agricole en Méditerranée qui permette à la fois la rencontre interactive entre disciplines, mais aussi un échange extrêmement fructueux avec ceux qui en ont la charge gestionnaire (ASA, Agence de l'eau, Services territoriaux, Chambres d'agriculture, Associations d'usagers,...). Plus de 70 communications et posters ont permis de débattre sur les transformations contemporaines au Nord et au Sud de la Méditerranée des multiples usages dont est l'objet l'eau agricole. Parmi eux, une vingtaine de présentations de chercheurs espagnols travaillant dans différentes universités, Girona, Barcelona, Granada, Murcia, Valladolid, Sevilla, CastillaLa Mancha.

\footnotetext{
2 http://www.lped.org/actes-du-colloque-eauagricole/presentation.html.
}

L'eau agricole reste encore majoritairement vouée à l'activité agricole, mais avec la transformation des besoins économiques et le développement de la question environnementale, on assiste à une progressive prise en compte de nouveaux "services", comme la recharge de nappes phréatiques, le développement de la biodiversité, le maintien de biotopes favorables à la faune piscicole, ainsi qu'à une diversification des usages (arrosage de jardins potagers ou d'agrément pour les particuliers, d'espaces publics pour les collectivités territoriales, gestion des inondations,...) qui restent méconnus car peu en relation avec la fonction première des canaux.

Au nord du bassin méditerranéen, leur existence même, du fait d'une très forte diminution de la population agricole, a été parfois oubliée, voire sacrifiée au profit d'une extension urbaine, et se pose aujourd'hui la question de leur prise en charge collective. Dans les pays du sud de la Méditerranée, les changements sociaux et la transformation des techniques d'irrigation agricole semblent de moins en moins appropriés à la disponibilité de la ressource et à la dégradation des sols qui entraîne une déstructuration de l'ancienne organisation sociale (en particulier dans les oasis et dans les zones péri-urbaines) et de fait de la gestion sociale de l'eau qui lui était attenante.

Quatre grandes thématiques ont jalonné les débats de ce colloque :

\section{De l'irrigation à la gestion durable de l'eau}

Les contributions présentées au cours de cet atelier ont permis de souligner les difficultés à envisager une gestion durable de l'eau agricole au regard de la multiplication d'usages non agricoles et des intérêts économiques et sociaux en jeu dans son utilisation. De façon transversale à l'ensemble des pays représentés, la question de la modernisation de l'irrigation agricole 
s'accompagne d'une recherche d'efficacité quant à l'utilisation de la ressource. Cette efficacité, essentiellement orientée vers la productivité agricole est cependant interrogée aujourd'hui au regard de ses conséquences écologiques. En Espagne, Italie et France, le cadre communautaire impose de nouveaux impératifs en termes d'économies d'eau qui se traduisent dans la plupart des cas par une modernisation des infrastructures hydrauliques au détriment des techniques d'irrigation gravitaire caractéristiques des régions méditerranéennes. Dans les pays du Sud de la Méditerranée, les politiques de modernisation de l'irrigation agricole issues du modèle colonial (construction de barrages, forages dans la nappe fossile) a permis le développement d'une agriculture productiviste fortement consommatrice d'eau et qui s'accompagne d'une disparition progressive des canaux d'irrigation traditionnel.

Dans un contexte où la préservation de la ressource en eau devient un enjeu majeur et la question de son partage entre des usages parfois contradictoires, une source renouvelée de conflits, la modernisation de l'irrigation agricole est présentée comme un moyen d'améliorer tout à la fois l'efficacité productive et environnementale de la gestion de l'eau agricole. Le questionnement de ce modèle de modernisation a fait l'objet de nombreuses contributions, interrogeant les conséquences écologiques, sociales et culturelles de la " disparition des canaux ". Dans un contexte méditerranéen, la persistance de techniques d'irrigation gravitaire consommatrice d'une quantité d'eau importante peut être perçue comme favorisant un " gaspillage " de l'eau. Les études de cas présentées, que ce soit en Espagne, en Italie ou en Tunisie et au Maroc soulignent pourtant comment les techniques gravitaires ne peuvent être réduites à leur seul usage productif mais doivent être considérées au regard du rôle qu'elles remplissent du point de vue écologique (recharge de nappes phréatiques, retours indirects au milieu), culturel (paysages de l'irrigation) et social (droits et formes de gestion locales associées à l'entretien des canaux). Les exemples développés ont tenté de montrer comment, face à ce constat, pouvait s'envisager des modèles de gestion durable des canaux à même d'intégrer la complexité écologique, économique et social qu'ils représentent.

Un premier élément de réponse porte sur l'échelle spatiale à laquelle doit être envisagée cette gestion. Cela nécessite d'appréhender la gestion de la ressource non plus uniquement à l'échelle locale ou globale, mais de replacer la question de l'efficacité de son utilisation à une échelle régionale, ou celle du bassin versant afin de ne plus considérer comme du "gaspillage " des techniques gravitaires qui permettent le maintien et la recharge de nappes supports à d'autres enjeux économiques et sociaux. Les débats ont porté sur les modèles de gestion à même de pérenniser les techniques gravitaires au regard des transformations économiques et sociales qui ont comme conséquence la multiplication des usages de l'eau agricole en France et en Italie, mais aussi de façon moins marquée en Espagne où l'activité agricole reste très majoritaire.

En Tunisie et au Maroc, la concurrence autour de l'utilisation agricole entre une paysannerie locale et le développement d'un agrobuisness pose des problèmes de marginalisation sociale et territoriale. L'exemple des Oasis du sud de la Tunisie et du Maroc témoigne des conséquences, non seulement écologiques, mais aussi sociales et culturelles des politiques de modernisation.

Les débats ont mis en exergue la nécessité de penser la gestion durable de l'eau agricole en tenant compte des dimensions sociales, temporelles, culturelles et économiques locales, mieux à même d'apporter des réponses en adéquation avec les enjeux du nécessaire partage de l'eau 
dans une région du monde particulièrement affectée par les crises hydriques.

\section{Les droits d'eau: propriété et appropriation symbolique}

L'accent a été mis ici plus spécifiquement sur les différents textes réglementaires nationaux, internationaux qui organisent la gestion de la ressource (LEMA, DCE,...), mais aussi les arrangements locaux qui organisent la complexité du partage entre les différents acteurs (Commission Exécutive de la Durance, collaborations interrégionales, internationales de frontières...). Ainsi en Tunisie, le défaut de régulation étatique ou participatif de l'eau a conduit à des initiatives de privatisation de l'eau, au détriment des paysans pauvres, qui deviennent de plus en plus exposés aux spéculateurs qui cherchent par tous les moyens à dominer les terres de ces derniers. En Europe, une cause récente de changement et générant des conflits est la loi sur l'Eau et les milieux aquatiques (LEMA) du 30 décembre 2006, qui impose pour 2014 une augmentation des débits réservés. Cette situation génère une crise par la nécessité de la réduction des prélèvements et donc des surfaces irriguées. La pertinence de la référence environnementale du 1/10ème du module annuel comme niveau d'étiage, a été discutée par les acteurs dans un contexte méditerranéen où les fortes précipitations hivernales ont une importante contribution à ce module et tendent ainsi à surestimer le débit de la rivière en dehors de ces pics.

Pour l'Italie, ont été abordées les perspectives sur la réglementation qui est en train de se mettre en place dans le cadre de la gestion participative des ressources en eau dans la région du Latium. Une loi cadre est rendue nécessaire du fait de l'utilisation croissante des cours d'eau et dans un objectif de trouver les meilleurs compromis entre le développement économique et la protection de l'environnement. Les débats ont porté sur l'approche participative dans le processus de planification, en application de la Directive Cadre sur l'Eau. Une loi cadre devrait être développée qui intégrerait 3 composantes :

- des mesures d'accompagnement pour la mise en œuvre de la planification

- des moyens pour financer la formation des professionnels

- financement des lieux de débat et de participation des acteurs

En Espagne, dans la région de Murcia, existent des formes collectives privées pour la distribution de l'eau qui est très rare dans cette région grâce à l'action d'un acteur clé: le répartiteur. Cette fonction créée en 1928 est très importante car le répartiteur fixe le prix du tour d'eau (à l'heure) en fonction de la demande et de la disponibilité de l'eau. Le répartiteur doit être un propriétaire mais pas un grand propriétaire, et être "fiable et bon". II est nommé par ses pairs.

Un système similaire existe dans les oasis notamment en Algérie. Dans les zones arides comme le Sahara l'eau a une grande importance et elle est considérée comme un bienfait de Dieu, qui donne et entretient la vie, purifie l'humanité et la planète. La Foggara l'unique moyen de captage des eaux à Timimoun en est le symbole. Comme l'eau est rare et précieuse, son usage et son partage le sont autant. Ce droit sacré de l'accès à l'eau, est le début d'une chaîne de sacralité, qui imprime l'urbain à Timimoun. La foggara impose une organisation de l'espace notamment avec des restrictions d'usages sur le passage des galeries. Les Foggaras ont été progressivement abandonnées avec la modernisation pour des causes diverses. Cependant un retour de ce type d'organisation est perceptible aujourd'hui dans différentes régions car elle correspond à un mode d'organisation parfois mieux adapté aux conditions locales et du fait de son importance sociale et culturelle. 
Finalement une discussion générale s'est engagée sur les modernités et les conflits perdurant et sur les traditions différentes dans les différents pays ou les régions qui ont vécu l'apparition de la modernité technique comme une colonisation. Cette modernité n'a souvent pas su intégrer les savoirs locaux ce qui a engendré des réactions sociales, des conflits et un manque de véritable solution technique durable. Les postures de modernisation opposant les modernes aux anciens étaient parfois trop simples et cachaient en réalité des jeux complexes d'intérêts et d'interdépendances.

\section{Biodiversité et paysages de l'eau}

Cet axe particulièrement novateur a recueilli un ensemble de présentations sur ce qu'on pourrait appeler les " services non marchands " de l'eau agricole et qui pourtant deviennent aujourd'hui centraux dans les nouvelles perspectives de gestion. En effet, cette eau, la plupart du temps véhiculée par des systèmes gravitaires a produit des paysages et une biodiversité spécifiques qui aujourd'hui sont considérés comme des plus-values sur les terrains concernés. De même a été abordée la question de la liaison entre eau de surface et eau souterraine qui reste centrale pour l'alimentation en eau potable de nombreuses populations.

Ces différents thèmes ont surtout été abordés par des chercheurs français et espagnols, la problématique environnementale touchant de manière moindre et différemment les pays du Sud de la Méditerranée pour lesquels la question de "l'appropriation" privée ou collective, reste l'enjeu essentiel. Néanmoins apparaissent des problèmes de déstructuration écosystémique, en particulier dans les oasis. Ainsi beaucoup d'oasis algériennes souffrent de l'accroissement important des débits utilisés pour l'irrigation, associés, surtout, à un usage souvent mal raisonné des res- sources hydriques. Ceci a conduit à des gaspillages importants, faisant apparaître des quantités non négligeables d'eaux excédentaires qui ont fortement perturbé les équilibres naturels dans les milieux urbains sahariens, milieux déjà très fragiles, caractérisés par des nappes superficielles très salées et souvent de faible profondeur et par des conditions topographiques qui ne facilitent pas l'évacuation des eaux usées. Ces milieux, devenus aujourd'hui malades de trop d'eau, avaient toujours fonctionné, auparavant, comme des systèmes hydrauliques fermés et bien équilibrés.

Les présentations des chercheurs espagnols ont beaucoup insisté sur "les paysages de l'eau " et leurs qualités patrimoniales risquant d'être remises en cause par les modernisations préconisées par le gouvernement espagnol. Des débats ont porté sur la nécessité du maintien d'un débit " écologique " minimum qui permette la conservation des valeurs naturelles, paysagères et des services environnementaux apportés par les eaux superficielles et la recharge des nappes aquifères. Les paysages de l'eau construits par les formes ingénieuses pensées aux siècles précédents tels que les foggaras en Algérie ou le système des terrasses en Provence ont été progressivement délaissées, oubliées, mais semblent aujourd'hui jouir $d$ 'un nouvel intérêt. Au-delà de l'aspect productif qu'ils avaient au siècle précédent, ils bénéficient d'un regain d'intérêt lié à la plus-value économique sur la valeur des terrains. Pour exemple en France du Sud, les terrains arrosés par la fraîcheur des canaux ou bénéficiant de terrasses en pierres sèches sont de plus en plus recherchés.

Enfin un autre aspect a suscité beaucoup d'intérêt dans les échanges, celui de la biodiversité spécifique créée par ces ouvrages hydrauliques. Par exemple a été abordée le rôle des petits canaux dans la circulation des poissons, et plus spécifi- 
quement le cas de la migration des anguilles dans le département du Var (France). Les canaux sont en effet des liens entre différents espaces aquifères et il est souvent oublié qu'ils contiennent une faune spécifique. Au siècle dernier, lorsque ces canaux étaient mis à sec pour réparation ou entretien, les tonnes de poissons qu'ils véhiculaient étaient tout " simplement " récupérés et mangés par les populations locales. Cette pratique, du fait de l'augmentation du niveau de vie et de la transformation des goûts des consommateurs qui privilégient le poisson de mer, a aujourd'hui disparu. Mais s'élèvent de nouvelles voix, comme celles des fédérations de pêche ou d'associations de protection de la nature pour demander une prise en compte différente de cette vie aquacole.

\section{Perspectives de gestion, participation et gouvernance}

Ce dernier axe a permis de faire le point sur les formes de gestion impulsées en réponse aux transformations économiques et sociales dans la gestion de l'eau agricole. Les cadres internationaux et nationaux de gestion intégrée de l'eau constituent aujourd'hui un modèle de " gouvernance " qui se fonde sur la participation et la concertation des “ communautés “ d'irrigants. II n'en résulte pas moins que leurs déclinaisons locales sont loin d'être uniformes. L'ensemble des communications proposées souligne ainsi l'importance de l'implication locale des acteurs dans leur mise en œuvre. Les réponses apportées s'expriment tantôt par des stratégies de résistances aux transformations voulues par le cadre législatif, tantôt au contraire par l'émergence ou le renforcement de certains groupes d'acteurs à même de transformer une injonction politique en innovations sociale, technique, économique.

Dans les pays du Maghreb, les conflits pour une ré-appropriation de sa gestion par la paysannerie sont encore très forts et visent à combattre une appropriation privée de celle-ci. Le caractère volontariste des politiques menées dans le secteur de l'irrigation agricole, héritage de la période postcoloniale, s'accompagne aujourd'hui de dispositif de participation visant à impliquer une paysannerie locale présentée par les pouvoirs publics comme réticentes aux innovations techniques. En Tunisie, la mise en place des GDA (Groupement de Développement Agricole) et au Maroc des AUEA (Association d'Usagers de l'Eau Agricole) sont l'expression d'une volonté d'impulser et d'impliquer localement les acteurs, en particulier les agriculteurs, dans une politique de modernisation technique et économique des modes de production agricole. Dans ces deux cas, les nouvelles associations d'irrigants se superposent aux organisations traditionnelles de gestion de la ressource qui assurent, suivant des modalités différentes parfois d'un périmètre à l'autre, la pérennité des droits d'eau, la répartition des tours d'eau, le règlement des conflits, la participation à l'entretien des aménagements hydrauliques. Dans ces modèles traditionnels, la gestion sociale de l'eau constitue un élément de structuration des communautés locales. Basée sur de petites propriétés foncières, dont le noyau est principalement la structure familiale, ce modèle est mis à mal du fait d'un ensemble de facteurs à la fois écologiques (épuisement de la ressource, sécheresse), mais aussi économiques (politique foncière de privatisation des terres, développement de grandes exploitations) et sociales (phénomène d'immigration, d'exode urbain). La juxtaposition de modèles de gestion sociale de l'eau, l'un communautaire, l'autre hérité du découpage administratif colonial, peut-elle donner lieu à la mise en place de nouvelles structures associatives qui peuvent apparaître aussi comme un moyen de palier les transformations sociales et économiques connues par les sociétés locales? Les 
réponses apportées par les intervenants de cet atelier sont contrastées. L'accompagnement de ces politiques publiques par une démarche participative et de formation auprès des agriculteurs peut permettre une appropriation locale des enjeux liés à une utilisation plus raisonnée de la ressource. Par exemple, au nord du Maroc, la mise en œuvre d'un plan de modernisation s'est accompagné d'un programme de formation qui a permis par le renforcement du rôle décisionnel des femmes dans la gestion de l'eau d'aboutir à une appropriation collective de ces nouvelles structures de gestion.

D'un autre côté, ces dispositifs participatifs peuvent aussi fragiliser l'organisation sociale qui est au fondement de la gestion collective de la ressource en eau. La tension entre une volonté de gestion collective de l'eau et un processus de privatisation de l'accès à la ressource constitue sans doute l'élément contradictoire des politiques actuellement menées.

Des débats très riches se sont centrés sur la place à accorder aux formes traditionnelles de gestion de l'eau dans un contexte de modernisation de l'irrigation. Sans tomber dans une mythification de l'organisation et de la dimension communautaire de ces sociétés locales, il s'agit plutôt d'appréhender la gestion communautaire comme le résultat de sociétés qui ont du et su s'adapter, à travers un rapport social, politique et technique, aux conditions climatiques et hydriques parfois très difficiles. Dans cette perspective, il s'agit de reconsidérer les savoirs locaux, non pas comme figés mais dans une approche dynamique, la modernisation et l'innovation devenant dans ce cadre non pas l'application d'un modèle, mais la réponse sociale locale à des changements structurels, économiques, politiques globaux. C'est en ce sens que la "résistance " des paysans du Sud du Maroc ou de la région de Sidi Bouzid en Tunisie a été analysée, non pas comme une " réticence " due à des " comportements archaïques ", mais comme un moyen de ré-appropriation de la gestion de la ressource et de résistances locales à des politiques nationales et internationales qui au nom de la modernité aboutissent à une paupérisation et une marginalisation de la petite paysannerie.

Au nord les situations sont très contrastées, avec des tentatives de gestion participative prometteuses en France (contrats de canaux) et des situations beaucoup plus conflictuelles pour l'Espagne, pays qui a choisi de favoriser une agriculture irriguée intensive au détriment d'une approche globale d'unité de la ressource. En Espagne, les terres irrigables valenciennes ont expérimenté un changement technologique sans précédents qui a eu d'importantes conséquences pour les communautés d'irrigants et les usagers directs de l'eau. La comparaison de deux communautés locales a permis de rendre compte des formes contrastées d'application de nouveaux modèles de gestion de l'eau agricole. Dans le cas des irrigants de Monforte del Cid, où la réduction de consommation est à peine significative, la question de l'accompagnement et de la participation des agriculteurs apparait comme un facteur clé pour aboutir à une gestion plus efficiente. Ces aspects, jusqu'à ce jour, n'ont pas été considérés par les administrations publiques, plus préoccupées par le financement du changement technologique que par l'amélioration de la gestion.

Les contrats de canaux, mis en place par l'Agence de l'eau Rhône Méditerranée Corse en France apparaissent comme des dispositifs innovants de gestion participative de l'eau agricole en région méditerranéenne. Basés sur le modèle des contrats de rivière, l'objectif des contrats de canaux est d'aboutir à un renouvellement de la gestion des aménagements hydrauliques gravitaires au regard des services qu'ils rendent aux territoires qu'ils traversent. Face à une diminution du 
nombre d'agriculteurs, la démarche participative engagée doit permettre l'implication de nouveaux acteurs (urbains, collectivités locales, fédération de pêche, association de protection de la nature) dans les conseils syndicaux qui gèrent les canaux.

Les débats auxquels ont donné lieu les expériences présentées, ont souligné le rôle central que peuvent encore jouer les agriculteurs dans la pérennité des canaux d'irrigation.

\section{Cita del artículo}

ASPE, Chantal y JACQUÉ, Marie. La durabilité de l'eau agricole en Méditerranée à l'épreuve des enjeux sociétaux contemporains. Hábitat y sociedad, 2011, № 2, p. 179-186.

$<w w w . h a b i t a t y s o c i e d a d . u s . e s>$.

http://dx.doi.org/10.12795/HabitatySociedad.2011.i2.09 